\title{
Hybrid microscopic depletion model in nodal code DYN3D
}

\author{
Y. Bilodid ${ }^{1,2}$, D. Kotlyar ${ }^{2}$, E. Shwageraus ${ }^{2}$, E. Fridman ${ }^{1}$, S. Kliem ${ }^{1}$ \\ ${ }^{1}$ Helmholtz-Zentrum Dresden-Rossendorf, \\ Bautzner Landstraße 400, 01328 Dresden, Germany \\ Tel.: +493512602020 \\ Fax: +493512603299 \\ y.bilodid@hzdr.de \\ ${ }^{2}$ Department of Engineering \\ University of Cambridge, CB2 1PZ Cambridge, United Kingdom
}

Keywords:

History effects; spectral history; microscopic depletion; DYN3D;

\section{Highlights}

- A new hybrid method of accounting for spectral history effects is proposed.

- Local concentrations of over 1000 nuclides are calculated using micro depletion.

- The new method is implemented in nodal code DYN3D and verified.

\begin{abstract}
The paper presents a general hybrid method that combines the micro-depletion technique with correction of micro- and macro- diffusion parameters to account for the spectral history effects. The fuel in a core is subjected to time- and space-dependent operational conditions (e.g. coolant density), which cannot be predicted in advance. However, lattice codes assume some average conditions to generate cross sections (XS) for nodal diffusion codes such as DYN3D. Deviation of local operational history from average conditions leads to accumulation of errors in XS, which is referred as spectral history effects. Various methods to account for the spectral history effects, such as spectral index, burnup-averaged operational parameters and micro-depletion, were implemented in some nodal codes. Recently, an alternative method, which characterizes fuel depletion state by burnup and ${ }^{239} \mathrm{Pu}$ concentration (denoted as Pu-correction) was proposed, implemented in nodal code DYN3D
\end{abstract}


and verified for a wide range of history effects. The method is computationally efficient, however, it has applicability limitations.

The current study seeks to improve the accuracy and applicability range of $\mathrm{Pu}$-correction method. The proposed hybrid method combines the micro-depletion method with a XS characterization technique similar to the Pu-correction method.

The method was implemented in DYN3D and verified on multiple test cases. The results obtained with DYN3D were compared to those obtained with Monte Carlo code Serpent, which was also used to generate the XS. The observed differences are within the statistical uncertainties. 


\section{Introduction}

Nodal diffusion codes such as DYN3D (Grundmann et al., 2005) are routinely used for nuclear reactor analyses. These codes obtain diffusion parameters of coarse-mesh space elements (nodes) from a library of homogenized few-group macroscopic reaction cross sections (XS), which are generated using lattice neutron transport codes. XS-libraries include dependence of diffusion parameters on operating conditions such as fuel temperature, moderator density, boron concentration and fuel burnup. Changes in fuel nuclide content during burnup are simulated by lattice codes in a depletion calculation under core-averaged (nominal) conditions. However, the fuel burnup in a specific node location in the core is accumulated under operating conditions (and thus neutron spectrum) that could be significantly different from the core average ones.

The history of local neutron spectrum, which deviates from the core- and cycle-averaged conditions used in lattice calculation, affects the fuel nuclide content and thus local XS. Spectral history is taken into account by various methods: micro-depletion (Ida and Tahara, 2000; Bahadir et al., 2005), spectral indexes (Baturin and Vygovskii, 2001) and exposureweighted operational parameters (Bahadir et al., 2005).

The micro-depletion is the most general method among all mentioned since it relies on the first principles while other methods utilize approximations, which limit their accuracy and range of applicability. The idea of microscopic depletion method is to calculate actual concentrations of important nuclides in each spatial depletion zone (node) and explicitly add their contributions to the homogenized macroscopic absorption and fission XS. However, the dependence of scattering matrix as well as microscopic XS on spectral history cannot be treated in the same way, while it is essential for accurate simulations.

The micro-depletion method is implemented in some nodal codes such as SIMULATE (Bahadir et al., 2005) and ANC (Ida and Tahara, 2000). To overcome limitations of microdepletion method, micro- and macro-XS are additionally parametrized against exposureweighted operational parameters (SIMULATE) or spectral history index (ANC). The depletion calculations in these codes track $\sim 50$ nuclides using simplified transmutation chains.

Recently, an alternative method was developed and implemented in DYN3D. This method accounts for the spectral history effect using so-called Pu-indicator correction (Bilodid and Mittag, 2010). It is based on an observation that all major history effects are 
primarily linked to ${ }^{239} \mathrm{Pu}$ buildup in the fuel. Therefore, parametrizing XS library against

${ }^{239} \mathrm{Pu}$ concentration as a spectral history indicator and then tracking local ${ }^{239} \mathrm{Pu}$ concentrations in nodal code was shown to produce accurate results for a wide range of spectral conditions (Bilodid et al., 2015). The Pu-indicator correction is applied not only to all macroscopic XS (e.g. scattering, diffusion, absorption and others) but also to kinetic parameters needed for time-dependent transient calculations (e.g. delayed neutron fractions). In some specific cases however, such as startup after long outage period or modelling systems with nonconventional fuels such as thorium, basic assumption of the Pu-indicator is not valid and therefore fails to produce accurate results.

This work aims at developing a general method that could be universally applied to different systems with alternative fuel types with the same accuracy as was demonstrated for LWR systems with $\mathrm{UO}_{2}$ fuel. The proposed here hybrid approach combines

- fast and flexible depletion solver, capable of tracking a full set of nuclides, with

- correction of absorption and fission macroscopic-XS using micro-depletion method and

- correction of other macroscopic-XS and all microscopic-XS using history indicator.

This paper describes the method, provides results of the verification tests, and explores sensitivity of the accuracy of the results to the number of nuclides taken into account in micro-depletion calculations. The paper is structured as follows. Section 2 briefly presents the computer codes used in the study. Section 3 describes the test case models. Section 4 describes the micro-depletion method, correction approach, and the hybrid micro-depletion method implemented in DYN3D. Section 5 reports the results of verification analysis. Section 6 summarizes the current work and presents a set of conclusions.

\section{Computer Codes}

The analyses reported here were performed with Serpent (Leppänen et al., 2015) and DYN3D (Grundmann et al., 2005). Serpent was used to generate lattice cross section data, which was then transferred to DYN3D to test the hybrid methodology. Serpent was also used to provide reference solutions for examined cases. 


\subsection{DYN3D}

DYN3D is a 3D nodal reactor dynamic code developed at Helmholtz-Zentrum DresdenRossendorf mainly for transient, but also for steady-state and fuel cycles analysis of LWR cores with hexagonal or square fuel assemblies.

DYN3D couples neutronic solution with thermal hydraulic and fuel rod models. Neutronic solver relies on nodal expansion method to solve multi-group diffusion or SP3 equation in rectangular, hexagonal or trigonal geometries, with pin-power reconstruction capabilities. Thermal hydraulic solver simulates single or two-phase flow in parallel channels, coupled by core mass flow or pressure drop as boundary conditions. Four-equation model is used to obtain coolant temperature and density distributions, heat fluxes, heat transfer regimes and DNBR. The model includes thermal properties of water-steam as well as helium and liquid sodium coolants. Fuel rod model is used to obtain fuel temperature profiles and includes burnup-dependent thermal properties of uranium dioxide.

The range of DYN3D applicability is greatly extended by multi-physics coupling with system codes such as ATHLET (Grundmann et al., 1995) and RELAP (Kozmenkov et al., 2007), CFD code ANSYS CFX and FLICA4 (Kliem et al., 2011), sub-channel thermal hydraulics SUBCHANFLOW (Gomez-Torres et al., 2012) and fuel performance code TRANSURANUS (Holt et al., 2015).

\subsection{Serpent}

Serpent is a continuous energy MC neutron transport code with burnup capabilities developed at research center VTT in Finland. This code allows modeling of complicated three-dimensional geometries. The code was developed as an alternative to deterministic lattice physics codes for generation of homogenized multi-group material properties (XS) for reactor analyses using nodal diffusion codes. The current version of Serpent includes libraries based on JEF-2.2, JEFF-3.1, ENDF/B-VI.8 and ENDF/B-VII evaluated data files.

The code has a number of features that dramatically reduce CPU time required for its execution, among them unified energy grid (Leppänen, 2009) and the use of Woodcock deltatracking (Leppänen, 2010) of particles. Serpent also has a built-in fuel depletion solver (Pusa and Leppänen, 2010) that is based on Chebyshev rational approximation (CRAM) method.

In this work, Serpent with JEFF-3.1 cross section library was used to obtain reference solutions for the test cases examined here and to generate homogenized macro and microscopic cross sections for DYN3D. 


\section{Description of Test Cases}

The performance of different spectral history correction methods was tested on two fuel cell models presented schematically in Fig. 1:

- BWR UOX - a BWR fuel lattice unit cell with fuel pin diameter of $1.21 \mathrm{~cm}$, lattice pitch $1.87 \mathrm{~cm}$, and with $3 \%{ }^{235} \mathrm{U}$ enriched uranium dioxide (UOX) fuel;

- PWR MOX - a PWR mixed oxide (MOX) fuel lattice unit cell with fuel pin diameter of $0.805 \mathrm{~cm}$, lattice pitch of $1.265 \mathrm{~cm}$, and with $4.4 \%$ total $\mathrm{Pu}$-content in heavy metal.

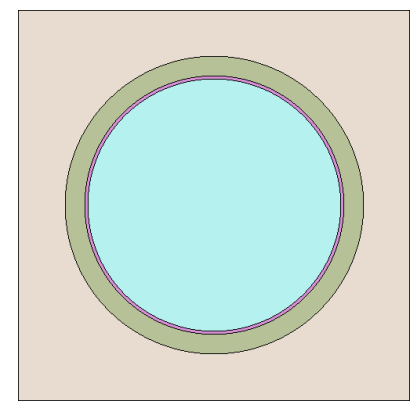

Fig. 1 Fuel cell model

Reflective boundary conditions were applied in all direction. The reference solution as well as the homogenized two-group XS for DYN3D were prepared using Serpent. The nominal conditions used for BWR fuel depletion were taken as follows: water density $\rho=400$ $\mathrm{kg} / \mathrm{m}^{3}$, fuel temperature $\mathrm{T}_{\mathrm{f}}=900 \mathrm{~K}$ and power density of $55 \mathrm{~W} / \mathrm{cm}^{3}$; the nominal conditions for PWR fuel depletion were: water density $\rho=700 \mathrm{~kg} / \mathrm{m}^{3}$, fuel temperature $\mathrm{T}_{\mathrm{f}}=900 \mathrm{~K}$ and power density of $100 \mathrm{~W} / \mathrm{cm}^{3}$.

\section{Implementation of micro-depletion in DYN3D}

This chapter presents the existing and currently suggested methods to account for spectral history effects. Section 4.1 presents the original method adopted in DYN3D. This section also presents an example in which the original method fails to accurately predict the core neutronic parameters, namely, a simulation of system restart after a long shutdown period. Section 4.2 describes the general micro-depletion problem and presents the method that was adopted in DYN3D. The weak points of the general micro-depletion correction are described in section 4.3. More specifically, the impact of lacking the scattering and microscopic cross 
sections corrections on the accuracy is demonstrated. The general hybrid method adopted in our analysis is described in section 4.4. Finally, section 4.5 presents some insights into the problem of choosing an optimal number of nuclides required for an efficient correction.

\subsection{Pu-indicator model in DYN3D}

Originally, DYN3D utilized the ${ }^{239} \mathrm{Pu}$-indicator method (Bilodid and Mittag, 2010) to account for the effect of spectral history on fuel neutronic properties. Nodal cross sections are corrected using local ${ }^{239} \mathrm{Pu}$ concentration by applying Eq.(1):

$$
\Sigma^{\text {corrected }}=\Sigma^{S A} \cdot\left[1+k\left(\sqrt{\frac{N_{P u}^{\text {actual }}}{N_{P u}^{\text {nominal }}}}-1\right)\right]
$$

where

$$
\begin{array}{ll}
\Sigma^{S A} & \text { is the nodal macroscopic cross section interpolated from XS-library, } \\
N_{P u}{ }^{\text {nominal }} & \text { is the concentration of }{ }^{239} \mathrm{Pu} \text { in the nominal depletion, } \\
N_{P u}{ }^{a c t u a l} & \text { is the actual local }{ }^{239} \mathrm{Pu} \text { concentration calculated by the nodal code, } \\
k & \begin{array}{l}
\text { is the historical coefficient, i.e. the proportionality factor between the } \\
\text { difference in }{ }^{239} \mathrm{Pu} \text { concentration and the difference in macroscopic cross } \\
\text { section. }
\end{array}
\end{array}
$$

Historical coefficients are obtained from the results of two lattice code depletion calculations; namely, nominal depletion used for XS-library preparation (under core- and cycle-averaged conditions) and off-nominal depletion (with deviation of one or few operational parameters). The Pu-indicator method was shown to accurately account for moderator density, fuel temperature and boron acid concentration history effects, as demonstrated in (Bilodid and Mittag, 2010; Bilodid et al., 2012, 2015).

Recent studies (Bahadir, 2014) demonstrated that long (few-years) outage has a significant effect on reactivity following start-up. The so-called Shutdown Cooling (SDC) reactivity is introduced in the fuel during few-year outages mainly as a result of decay of fissile ${ }^{241} \mathrm{Pu}$ into absorbing ${ }^{241} \mathrm{Am}$ and buildup of ${ }^{155} \mathrm{Gd}$ from its precursor ${ }^{155} \mathrm{Eu}$.

The outage was simulated using BWR fuel pin model (see section 3). The chosen powerdepletion history is as follows: 1) burnup under nominal conditions until $30 \mathrm{MWd} / \mathrm{kgHM}$; 2) 4 years outage and 3) burnup under nominal conditions up to $50 \mathrm{MWd} / \mathrm{kgHM}$. Deviation of DYN3D results from Serpent reference solution is shown in Fig. 2. 


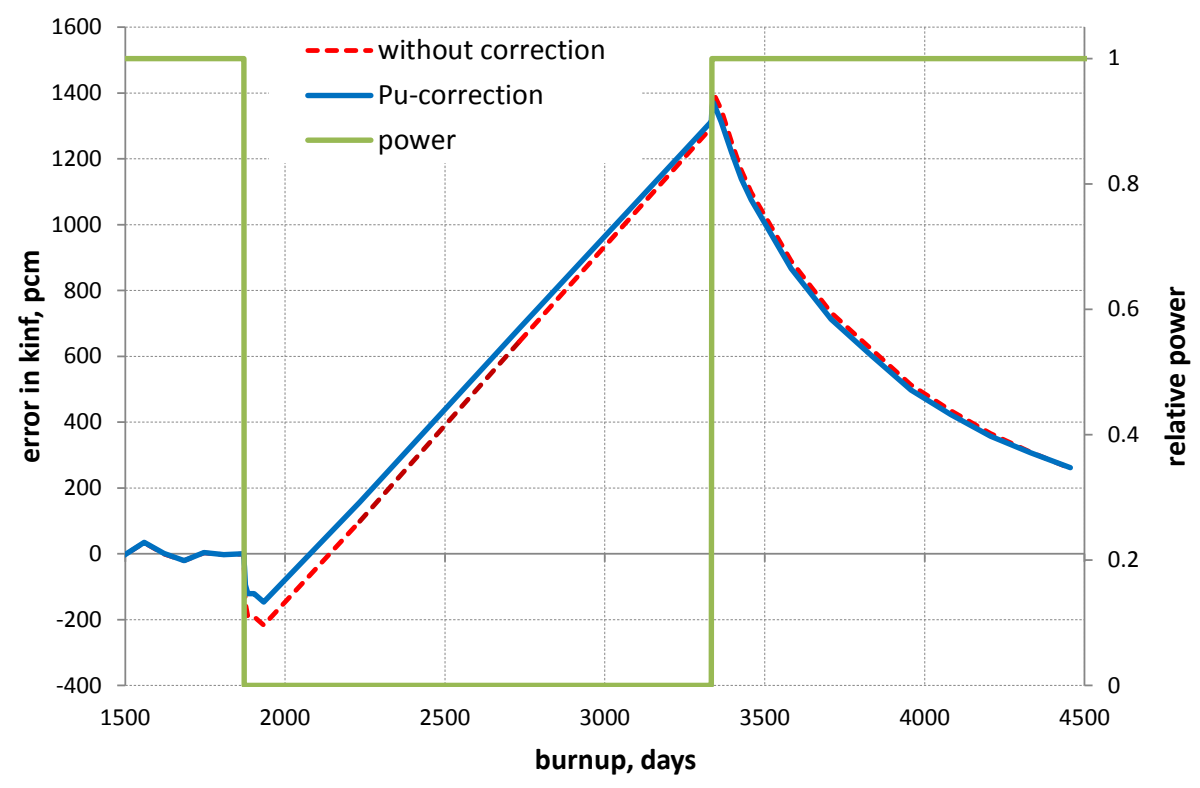

Fig. 2 Difference in reactivity for variable power history

As can be observed from Fig. 2, Pu-correction alone fails to reproduce reactivity during shutdown and subsequent burnup period. ${ }^{239} \mathrm{Pu}$ concentration increases slightly at the beginning of the outage due to decay of ${ }^{239} \mathrm{~Np}$ and then stays constant due to its long half-life time $\mathrm{T}_{1 / 2}=24100$ years. Therefore, the change in ${ }^{239} \mathrm{Pu}$ concentration does not reflect the changes in fuel properties and Eq.(1) fails to reproduce correct XS.

\subsection{General micro-depletion approach}

While homogenized macroscopic XS for nodal codes such as DYN3D are usually prepared by the lattice codes, the spatial distribution of the most important fission product poisons such as ${ }^{135} \mathrm{Xe}$ and ${ }^{149} \mathrm{Sm}$ are calculated within the nodal code. This is done by solving (simplified) depletion chains in each node using nodal fluxes and homogenized microscopic cross section prepared by the lattice code. Poisoning contribution is taken into account by socalled micro-depletion correction:

$$
\Sigma=\Sigma^{S A}+\sum_{i} \sigma_{i}\left(N_{i}^{\text {actual }}-N_{i}^{S A}\right)
$$

where,
$i \quad$ is the isotope index which represents ${ }^{135} \mathrm{Xe}$ and ${ }^{149} \mathrm{Sm}$,
$\Sigma^{S A} \quad$ is a homogenized macroscopic absorption XS calculated by the lattice 
code in a single assembly model,

$\sigma_{i} \quad$ is the homogenized microscopic absorption XS of nuclide $i$,

$N_{i}^{S A} \quad$ is the poison concentration calculated by the lattice code,

$N_{i}^{\text {actual }}$ is the local poison concentration calculated by the nodal code.

In DYN3D, simplified ${ }^{135} \mathrm{I}->{ }^{135} \mathrm{Xe}$ and ${ }^{149} \mathrm{Pm} \rightarrow{ }^{149} \mathrm{Sm}$ chains are used to model the poisoning effects during the fuel cycle and to simulate xenon transients (e.g. control rodinduced oscillations).

The physical reason behind spectral history effects is the deviations in local fuel nuclide content from the content predicted in XS-library generation by lattice code. Therefore, introducing more nuclides in Eq.(2) allows to better account for the spectral history effect. This method is referred to as micro-depletion, since the local (within node) nuclide content should be calculated by applying a depletion solver.

In this work, a new micro-depletion solver was implemented in DYN3D. The local concentrations of about 1100 nuclides are tracked. About 300 nuclides with neutron interaction data are included in Eq.(2) to correct the macroscopic absorption and fission XS.

Few-group isotopic microscopic cross sections for reactions $(\mathrm{n}, \gamma),(\mathrm{n}, \mathrm{f}),(\mathrm{n}, 2 \mathrm{n}),(\mathrm{n}, 3 \mathrm{n})$ and $(n, \alpha)$ are generated using Serpent. The data was prepared using JEFF 3.1 evaluated data file.

For each node in DYN3D at each time step, the Bateman equations are solved by computing the matrix exponential (Eq. 3).

$\mathbf{N}(t)=e^{\mathbf{A} \Delta t} \mathbf{N}(0)$

where, $\mathbf{N}=\left[N_{1} \cdots N_{n}\right]$ is unique for a given time point and $N_{j}$ is the atomic density of nuclide $j$. The operator $\mathbf{A}$ in Eq.(3) represents the transmutation matrix which depends on the rates of neutron induced and decay reactions. Matrix $\mathbf{A}$ includes removal terms on the diagonal and production rates on off-diagonal positions as explained in Eq.(4).

$$
\begin{aligned}
& \mathrm{A}_{j, j}=-\lambda_{j}-\sum_{g} \sigma_{g, j} \phi_{g} \\
& \mathrm{~A}_{j, k \neq j}=\lambda_{k \rightarrow j}+\sum_{g} \sigma_{g, k \rightarrow j} \phi_{g}
\end{aligned}
$$


where $\lambda_{j}$ and $\sigma_{g, j}$ are the decay constant and few-group absorption cross section respectively of nuclide $j$, while $\lambda_{k \rightarrow j}$ and $\sigma_{g, k \rightarrow j}$ are the decay constant and the few-group cross section of nuclide $k$ which lead to nuclide $j$ respectively and $\phi_{g}$ is the neutron flux in group $g$.

The few-group microscopic XS for each nuclide are obtained by interpolation from the XS-library using beginning-of-step material temperatures. The beginning-of-step neutron flux and microscopic XS are used in the transmutation matrix (Eq. 4). They are considered to be constant during the depletion step, which in typical DYN3D burnup simulations is 5-10 days. Predictor-corrector or sub-step methods are not used in the current implementation, but will be considered in the future.

The method implemented in DYN3D to solve transmutation matrix exponential is Chebyshev rational approximation (CRAM) (Gonchar and Rakhmanov, 1989; Pusa, 2011). This method was proven to be particularly suitable (Pusa and Leppänen, 2010) for fuel depletion and radioactive decay problems.

\subsection{Sensitivity of scattering matrix and microscopic cross sections to spectral history}

Applicability of the correction given in Eq. (2) was tested on the BWR cell model. The tested pin cell was depleted under off-nominal water density $700 \mathrm{~kg} / \mathrm{m}^{3}$ condition up to $50 \mathrm{MWd} / \mathrm{kgHM}$. This case represents quite an extreme deviation of neutron spectrum from the nominal conditions (nominal water density equal to $400 \mathrm{~kg} / \mathrm{m}^{3}$ ). Ignoring the spectral history effects leads to an error build-up of $12000 \mathrm{pcm}$ in k-inf at $50 \mathrm{MWd} / \mathrm{kg}$ as shown in Figure 3. 


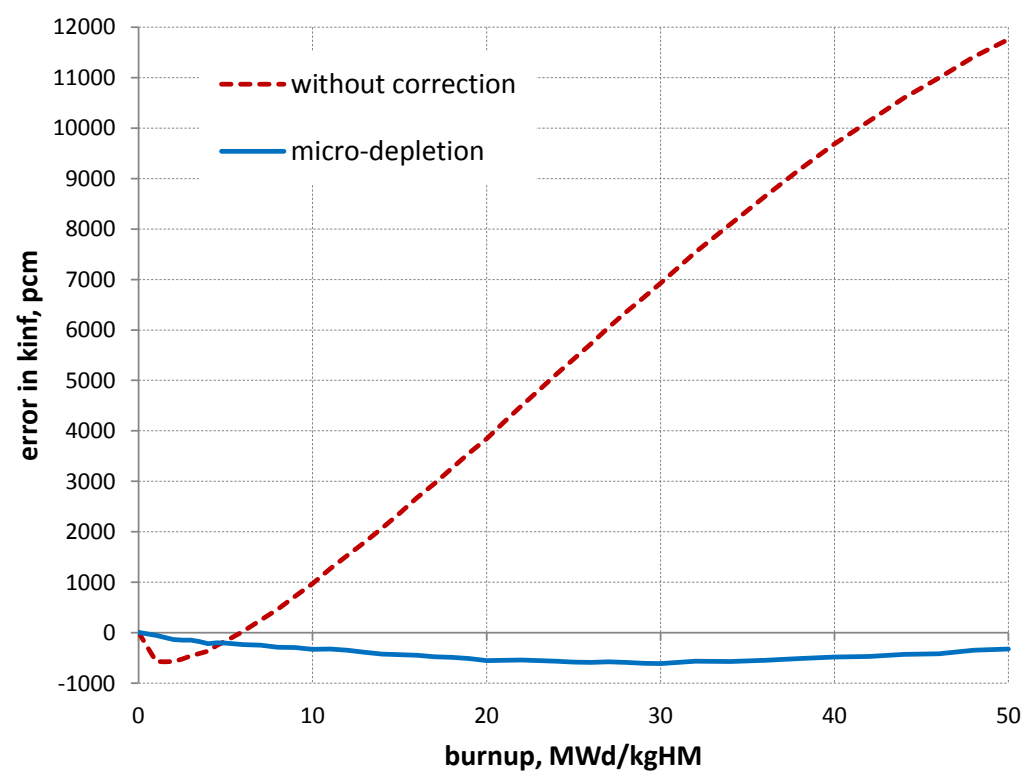

Fig. 3 Accuracy of the general micro-depletion method

The macroscopic cross section correction described by Eq. (2) includes concentrations of all nuclides with available XS data $(\sim 300)$, and accounts for most of the spectral history effect. Applying such correction indeed reduces the error from $12000 \mathrm{pcm}$ to $600 \mathrm{pcm}$. The remaining difference however is still not negligible. This error can be attributed to the dependence of scattering matrix components and homogenized microscopic XS on the fuel nuclide inventory and hence on spectral history.

Fig. 4a depicts the deviation of ${ }^{235} \mathrm{U}$ microscopic thermal absorption and fission XS from the reference ones. Relative difference in microscopic-XS for the same temperature but different spectral histories reaches about $20 \%$ at $50 \mathrm{MWd} / \mathrm{kgHM}$. The impact of spectral history on the down- and up-scattering in 2-group structure is shown in Fig. 4b. Although not presented here, preliminary test calculations have shown that expansion to 8-group structure significantly reduces the deviations in the scattering matrix (down to 5\%), but has minor impact on microscopic absorption cross section (the deviation is reduced from $20 \%$ in 2 groups to $15 \%$ in 8 groups). 


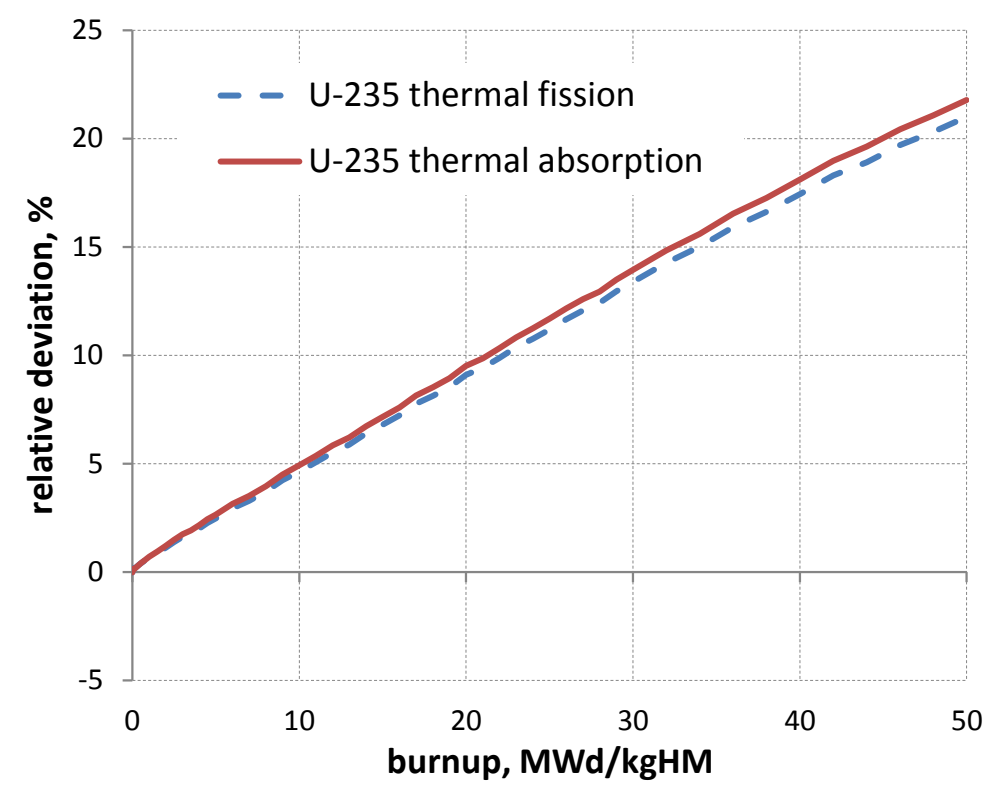

(a) ${ }^{235} \mathrm{U}$ microscopic $\mathrm{XS}$

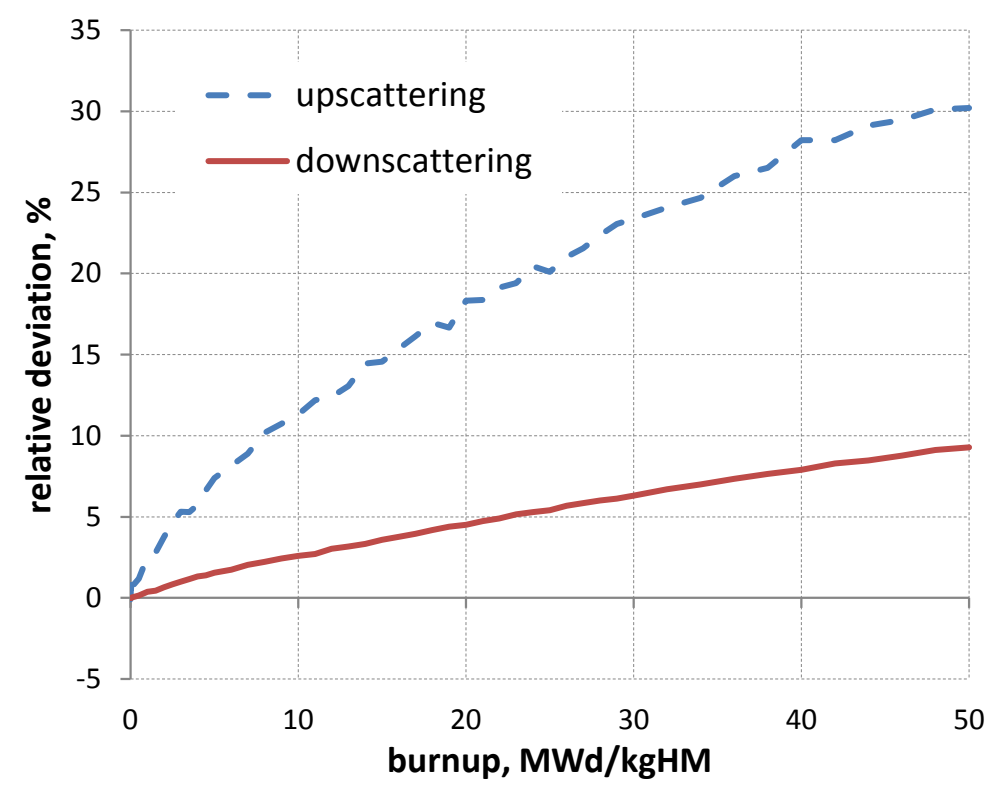

(b) Macroscopic scattering XS

Fig. 4 Relative difference in microscopic and macroscopic cross sections

As shown in Fig. 3 and Fig. 4, accounting for dependence of microscopic-XS and scattering matrix components on spectral history is essential for accurate representation of spectral history effects.

Nodal codes with micro-depletion methodology utilize various approaches to this problem. In CASMO/SIMULATE package, additional depletion calculations (histories) with variation of corresponding parameter are performed (historical moderator density, fuel temperature, boron acid concentration, control rod presence, etc.) and all macro- and micro- 
XS are functionalized against historical parameters (Bahadir et al., 2005). The ANC nodal code corrects microscopic-XS using burnup-averaged spectral index (Ida and Tahara, 2000).

\subsection{Hybrid micro-depletion method}

In this work, we propose to apply a different approach which adopts the correction shown in Eq. 1 and could be applied to any of the parameters, i.e. microscopic-XS, diffusion coefficients and scattering matrix. To make the method more general (including non-standard fuel types such as ${ }^{232} \mathrm{Th}^{233} \mathrm{U}$ ), a sum of all fissile isotope concentrations ${ }^{233} \mathrm{U},{ }^{235} \mathrm{U},{ }^{239} \mathrm{Pu}$ and ${ }^{241} \mathrm{Pu}$ is chosen as a representative spectral history indicator:

$$
\sigma^{\text {actual }}=\sigma^{S A}\left[1+k\left(\frac{N_{\text {fissile }}^{\text {actual }}}{N_{\text {fissile }}^{\text {SA }}}-1\right)\right]
$$

where

$$
\begin{array}{ll}
\sigma^{S A} & \text { is the nodal macroscopic cross section interpolated from XS-library, } \\
N_{\text {fissile }}^{S A} & \text { is the concentration of fissile nuclides in the nominal depletion, } \\
N_{\text {fissile }}^{\text {actual }} & \text { is the actual local fissile concentration calculated by the nodal code, } \\
k & \text { is the history coefficient. }
\end{array}
$$

The history coefficients $k$ are calculated for each nuclide and reaction type as a ratio between the history indicator (fissile content) and microscopic reaction XS obtained from two identical Serpent branching sets with nominal and the chosen off-nominal depletions:

$$
k=\delta \sigma /_{\delta N_{\text {fissile }}}=\frac{\sigma^{o f f}-\sigma^{\text {nominal }}}{\sigma^{\text {nominal }}} \frac{N_{\text {fissile }}^{\text {nominal }}}{N_{\text {fissile }}^{\text {off }}-N_{\text {fissile }}^{\text {nominal }}}
$$

Similarly to all macro- and microscopic XS, the history coefficients $k$ are tabulated in XS-library as function of burnup and operational parameters.

By applying the correction presented in Eq.(6), we assume linear proportionality between the value of history indicator and the correction factor. Validity of this assumption is demonstrated in Fig. 5, where relative deviations of different diffusion parameters (numerator in Eq.(7)) in different spectral histories are plotted versus deviation of the history indicator (denominator in Eq.(7)). Data points in Fig. 5 are calculated for a burnup $30 \mathrm{MWd} / \mathrm{kgHM}$ accumulated under different spectral conditions with variation in water density 400$700 \mathrm{~kg} / \mathrm{m}^{3}$ and fuel temperature $900-1500 \mathrm{~K}$. 


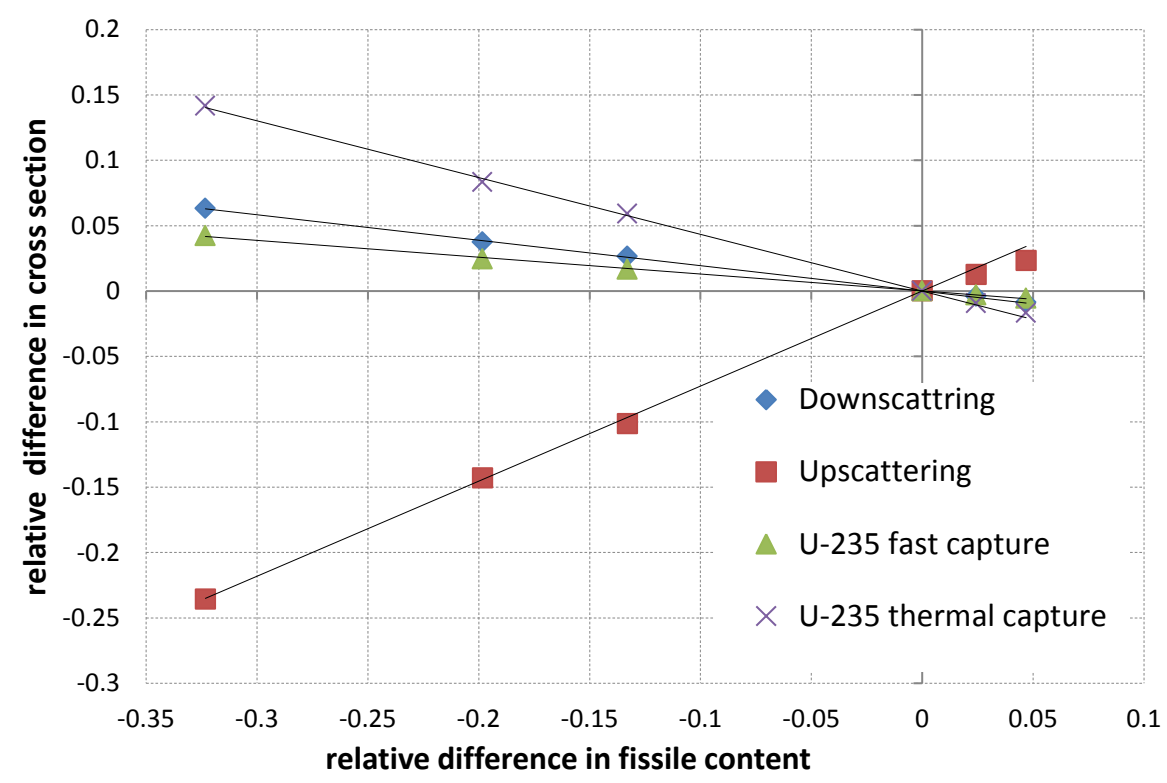

Fig. 5 Linear proportionality, $k$, to the fissile content

Taking into account the dependence of microscopic XS on spectral history transforms Eq. (2) into the following relation:

$$
\Sigma_{j}^{\text {actual }}=\Sigma_{j}^{S A}+\sum_{i}\left(\sigma_{j i}^{\text {actual }} N_{i}^{\text {actual }}-\sigma_{j i}^{S A} N_{i}^{S A}\right)
$$

The hybrid micro-depletion method implemented in DYN3D utilizes Eq.(8) to correct macroscopic absorption and fission XS, while correction (6) is applied on microscopic-XS, scattering matrix and diffusion coefficients.

For the consistency of nuclide densities, $N_{i}^{S A}$ in Eq.(8) as well as $N_{\text {fissile }}^{S A}$ in Eq.(6) are not taken from Serpent results but calculated by DYN3D for the nominal conditions in a single-assembly geometry. This eliminates the systematic error coming from the differences in DYN3D and Serpent depletion chains and flux solvers.

\subsection{Number of nuclides considered in the correction of XS}

The depletion solver and micro-depletion correction methodology implemented in DYN3D can be used with different number of nuclides. The size of the transmutation matrix (i.e. number of considered nuclides) has strong impact on the computation time, since the depletion problem should be solved for each region and each time step. However, in this study the nuclide inventory considered in all examined test cases (both in reference Serpent calculations and in DYN3D) was about 1100 nuclides, from which only 300 have neutron interaction data in JEFF-3.1. For the sensitivity analysis, these 300 nuclides were ranked 
according to their contribution to the correction (Eq. 8). The list of nuclides accounted in correction Eq. 8 was sequentially shortened (excluding the least important nuclides) to observe the effect on the results as shown in Fig. 6.

Majority of the nuclides do not contribute to the correction because their concentration is low. In the considered case, when the number of nuclides included in the correction procedure was reduced from 300 down to 80 , no notable impact on the results was observed. Further shortening to 50 nuclides kept the error in multiplication factor within $50 \mathrm{pcm}$ and shortening to 40 nuclides results in error of about $100 \mathrm{pcm}$. Further decrease in the number of accounted nuclides results in significant error.

It must be emphasized that in the current analysis, the entire set of nuclides (1100) was depleted in all cases, while a smaller subset of nuclides was used to correct the XS using Eq. 8. These results indicate that significant reduction in the number of nuclides to be followed is possible without losing the correction accuracy. Only about 50 nuclides actually affect the reactivity and knowing their exact concentrations is sufficient to account for the spectral history effects. However, for accurate tracking of these 50-80 most contributing nuclides, it is necessary to consider all intermediate steps in transmutation chains, which requires tracking a much larger (on the order of hundreds) number of nuclides.

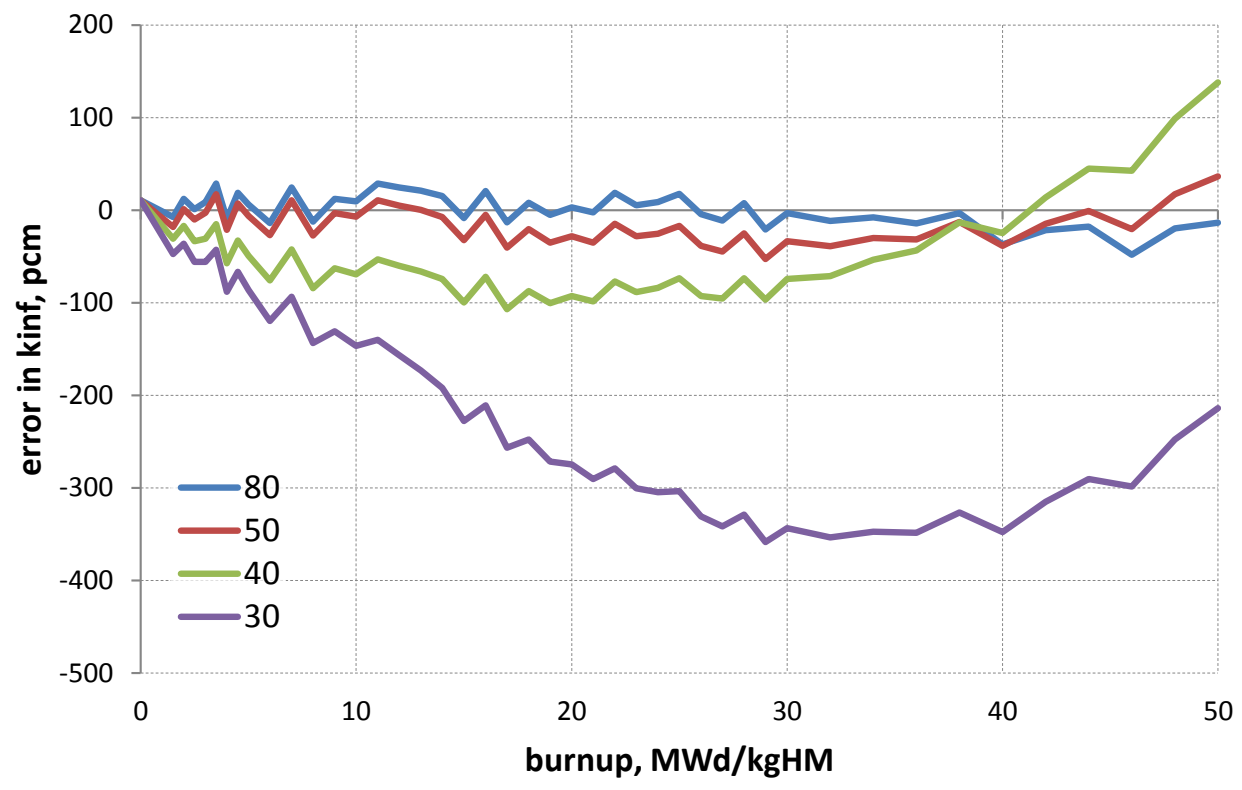

Fig. 6 Effect of the number of considered nuclides on accuracy.

The most computationally expensive operation in depletion solver is the calculation of transmutation matrix exponential by CRAM, and its performance depends strongly on the 
matrix size. Preliminary studies indicate that reducing the matrix size from $1100 \times 1100$ down to $350 \times 350$ decreases the CRAM calculation time of a single depletion step by a factor of $9^{1}$. However, detailed depletion matrix optimization studies (i.e. accuracy vs. matrix size) were not carried out here but are planned to be performed in the future.

It should also be mentioned that reduced nuclide inventory could be used for reactivity and power distribution analysis but could not be used for simulation of decay heat following a shutdown.

\section{Verification of the hybrid micro-depletion method}

In order to demonstrate the general applicability of the hybrid micro depletion method, it was tested on BWR and PWR fuel models (described in section 3) in various spectral conditions.

It should be noted that Monte Carlo results are always accompanied by statistical uncertainty, which propagates into DYN3D input (as XS-library). Fig. 7 illustrates the statistical noise up to $\pm 40 \mathrm{pcm}$ in deviation of DYN3D multiplication factor from the results obtained with Serpent. Therefore, any reactivity differences presented below that are smaller than this statistical noise should be considered as an indication of good agreement between tested methods. Neutron statistics used for the XS-library branching calculations and for the reference depletion calculation included $10^{5}$ neutron histories in 300 active cycles.

\footnotetext{
${ }^{1}$ The non-parallel CRAM calculation of $1100 \times 1100$ matrix takes about $0.18 \mathrm{sec}$ on Intel ${ }^{\circledR}$ Core ${ }^{\mathrm{TM}}$ i7 desktop $\mathrm{CPU}$
} 


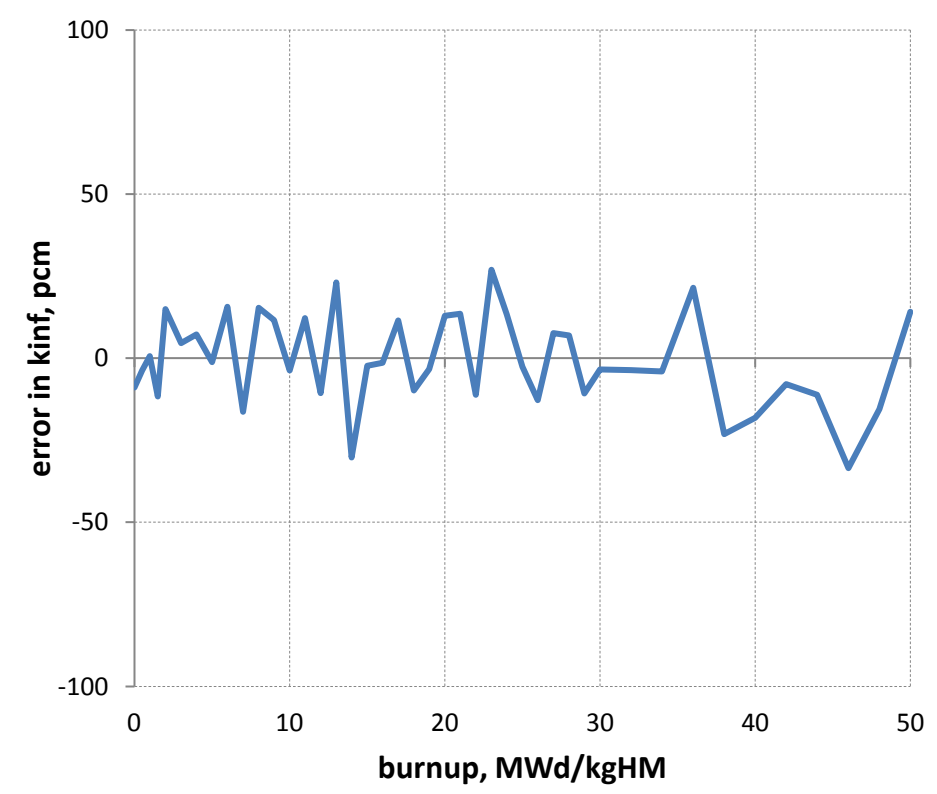

Fig. 7 Statistical noise in DYN3D and Serpent results.

The results for the BWR pin test case are presented in Fig. 8. In all the examined cases, DYN3D used the same library of the macroscopic and microscopic XS. Burnup-dependent nuclide content for Serpent branching calculations was calculated under nominal conditions: water density $400 \mathrm{~kg} / \mathrm{m}^{3}$ and fuel temperature $900 \mathrm{~K}$. The off-nominal water density 700 $\mathrm{kg} / \mathrm{m}^{3}$ was used to generate historical coefficients (Eq. 7) for micro-XS and scattering matrix. This set of data was used to simulate depletion with off-nominal water density $500 \mathrm{~kg} / \mathrm{m}^{3}$ (Fig. 8a), fuel temperature $1200 \mathrm{~K}$ (Fig. 8b) and combination of off-nominal water density $500 \mathrm{~kg} / \mathrm{m}^{3}$ with prolonged outage simulation (Fig. 8c).

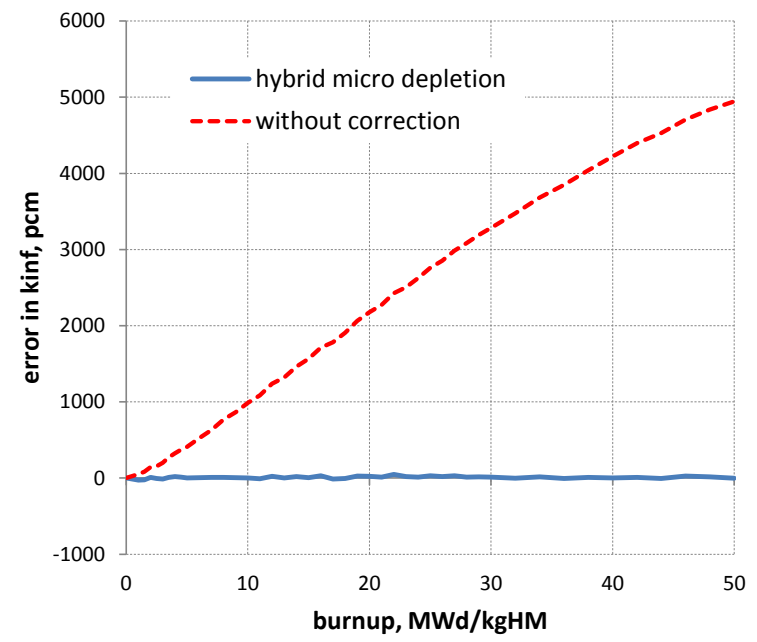

a) water density $500 \mathrm{~kg} / \mathrm{m}^{3}$

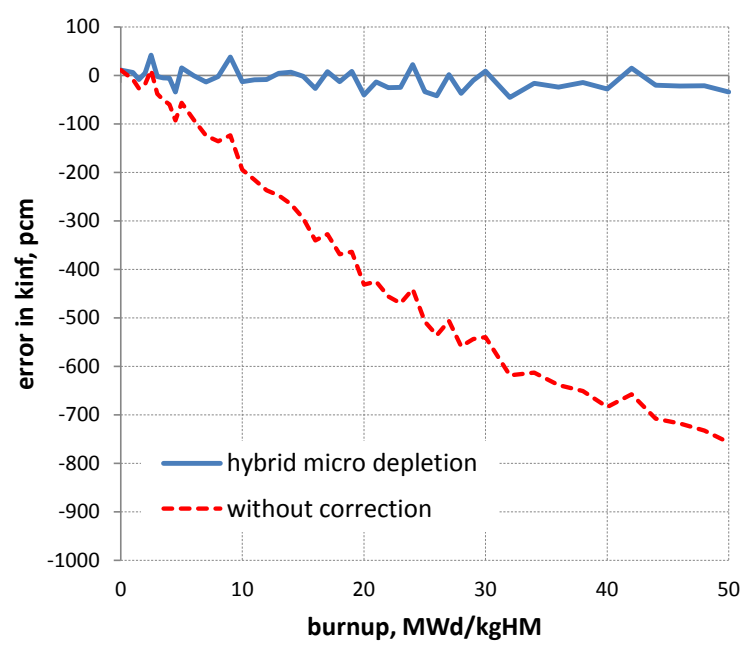

b) fuel temperature $1200 \mathrm{~K}$ 


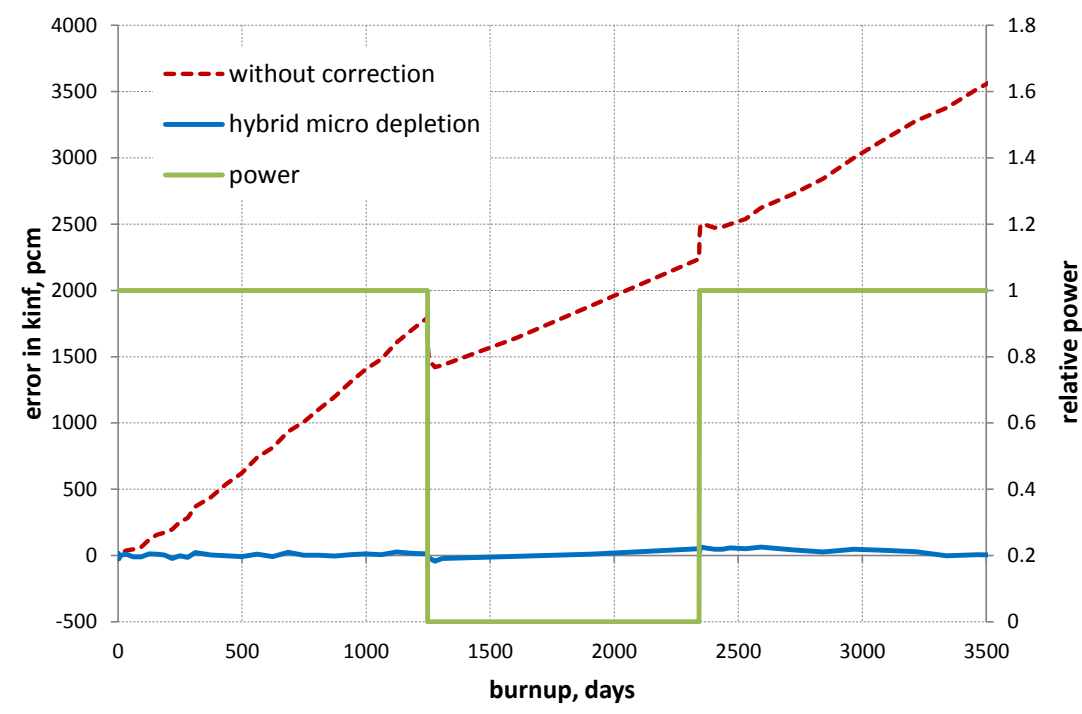

c) 3-years outage between two burnup periods

Fig. 8 BWR UOX results.

The results of PWR MOX pin test cases are shown in Fig. 9: case a) depletion with offnominal water density $600 \mathrm{~kg} / \mathrm{m}^{3}$; b) depletion with fuel temperature $1200 \mathrm{~K}$; c) boron concentration $500 \mathrm{ppm}$; d) combination of fuel temperature $1000 \mathrm{~K}$ with water density $750 \mathrm{~kg} / \mathrm{m}^{3}$; e) outage between two exposure periods with variation in power.

Nominal conditions used to generate PWR MOX XS-library were as follows: water density $700 \mathrm{~kg} / \mathrm{m}^{3}$, fuel temperature $900 \mathrm{~K}$ and $0 \mathrm{ppm}$ boron concentration. Off-nominal water density of $800 \mathrm{~kg} / \mathrm{m}^{3}$ was used to generate the historical coefficients. 


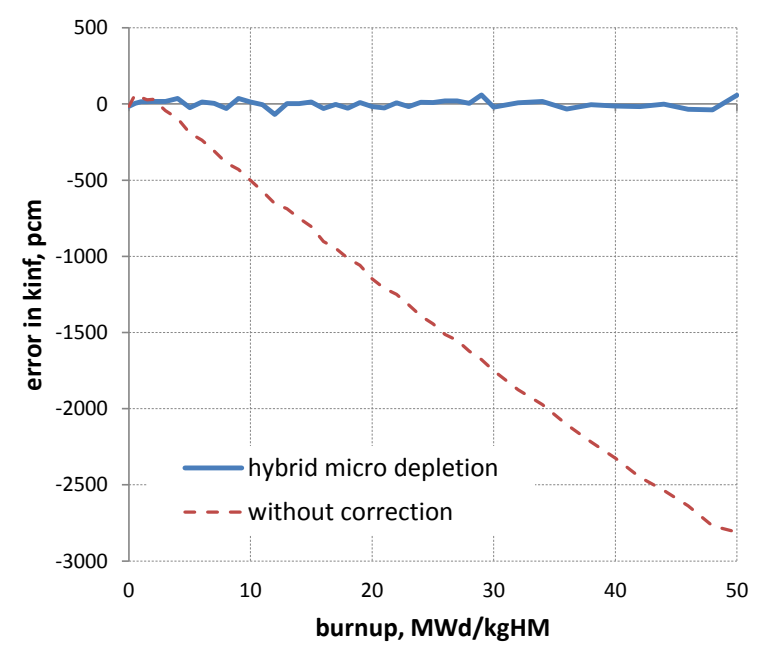

a) water density $600 \mathrm{~kg} / \mathrm{m}^{3}$

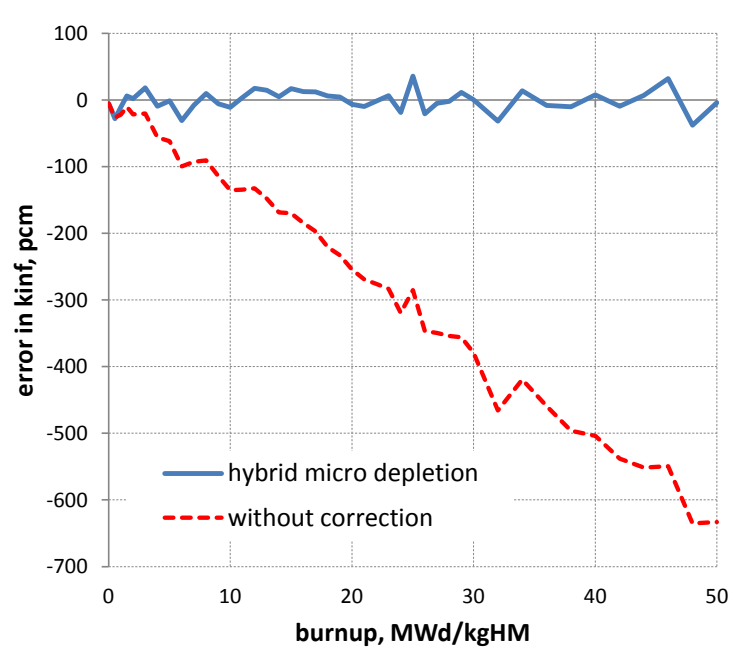

c) boron concentraion $500 \mathrm{ppm}$

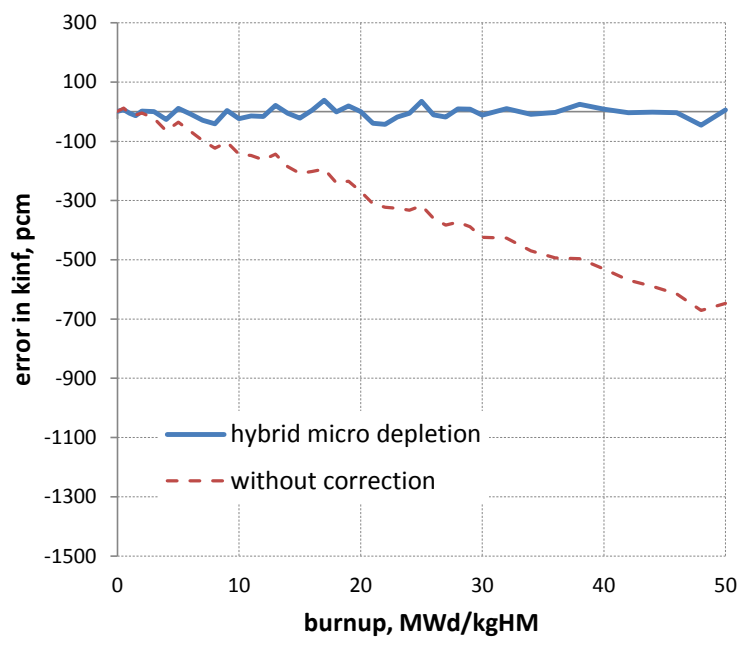

b) fuel temperature $1200 \mathrm{~K}$

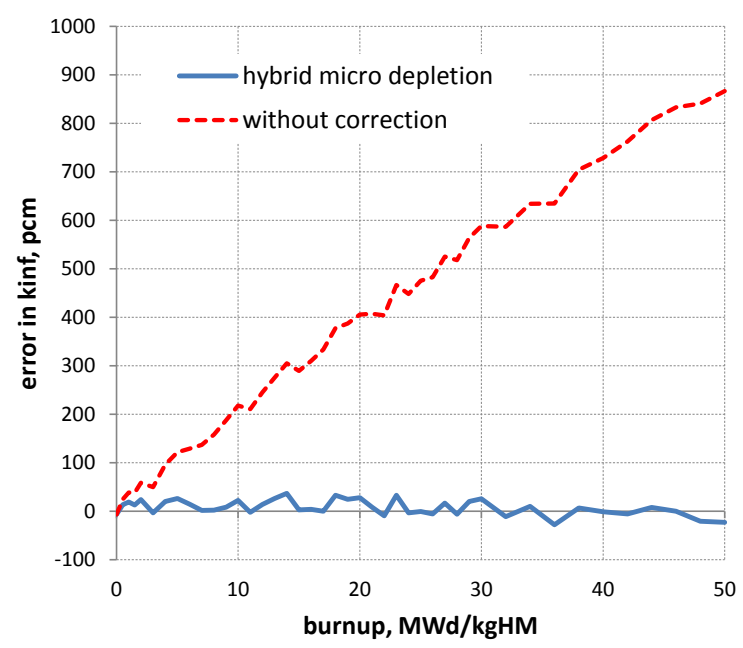

d) fuel temperature $1200 \mathrm{~K}$, water density

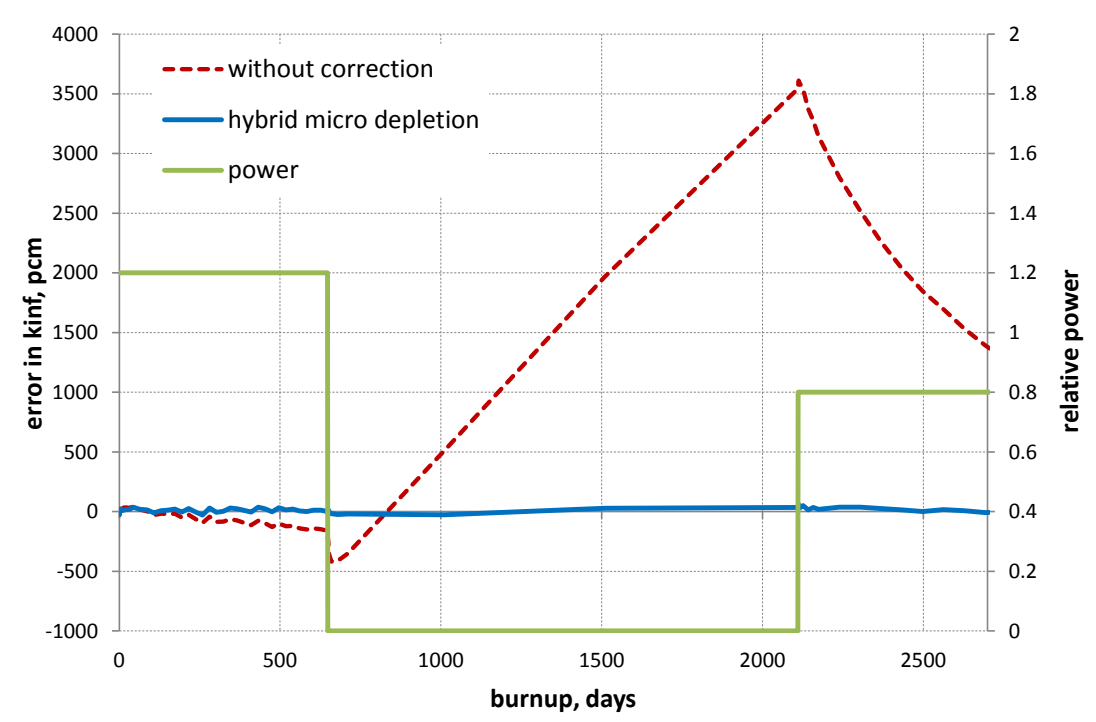

e) 4-years outage between two burnup periods

Fig. 9 MOX results. 
In all simulated test cases, the deviation of DYN3D multiplication factor from Serpent is within $50 \mathrm{pcm}$.

\section{Summary and conclusions}

Reactor dynamics simulators, such as DYN3D, require pre-generated homogenized neutronic parameters (cross sections - XS). These parameters are generated by lattice codes, in which fuel is depleted under core average (nominal) conditions. However, the local operational history affects the local nuclide content and thus neutronic properties (XS).

In order to account for local spectral history in each burnup region, nodal codes utilize various XS correction methods, which can be subdivided into two groups: history indicators and micro-depletion. The first group of methods correct the local XS proportionally to some spectral history indicator, which could be exposure-weighted operational parameters (moderator density, fuel temperature, control rod presence, etc.), exposure-weighted spectral index (e.g. thermal to fast flux ratio) or local concentration of ${ }^{239} \mathrm{Pu}$. These methods produce accurate results for some fuel types and history effects but fail in cases where the methods basic assumption is not applicable (e.g. outage simulation and alternative fuel compositions). The second group of methods explicitly takes into account the contribution of 30-50 most important nuclides to macroscopic absorption and fission XS, whose local concentrations are tracked explicitly for each node. The choice of considered nuclides as well as transmutation chains simplifications are case specific and should be reconsidered for each reactor type and fuel composition. Moreover, to produce accurate results, micro-depletion method needs to be supplemented with a correction to other macroscopic data (scattering, diffusion coefficients) and to microscopic XS. Such correction could be provided by one of the methods from the first group, so the resulting methodology is denoted as hybrid micro-depletion.

This paper proposes an alternative generalized hybrid method capable of accurately accounting for various operational conditions and outage history effects, which combines extended micro-depletion with Pu-indicator method. Macroscopic absorption and fission XS are corrected using the micro-depletion correction, where the detailed nuclide content (1-2 thousands of nuclides) is calculated for each node. Detailed nuclide content consideration allows obtaining concentrations of neutronically important nuclides without major approximations and simplifications in transmutation chains. Microscopic-XS as well as the scattering matrix, diffusion coefficients and other fuel properties are corrected proportionally 
to the sum of fissile nuclides ${ }^{233} \mathrm{U},{ }^{235} \mathrm{U},{ }^{239} \mathrm{Pu}$ and ${ }^{241} \mathrm{Pu}$ absorption, which is chosen as the history indicator.

The hybrid method was implemented in DYN3D. The depletion solver utilizes fast and accurate Chebyshev rational approximation (CRAM) method, which is particularly computationally efficient when applied to transmutation matrices.

The accuracy of the proposed hybrid micro-depletion method was verified against Serpent Monte-Carlo reference in 2D infinite lattice test cases with BWR UOX and PWR MOX fuel unit cells. For each fuel type, a library of two-group macroscopic-XS, isotopic microscopic-XS and historical coefficients was generated using two identical Serpent branching sets with different depletion histories. Each parameter in XS-library depends on burnup and instantaneous coolant density, fuel temperature and boron concentration for PWR cases. The obtained data sets were used by DYN3D to simulate fuel depletion in various spectral conditions (different from those used for the XS-library preparation), including outages. In all test cases, the deviation of DYN3D results with hybrid micro-depletion from the Serpent reference was within statistical uncertainty.

The presented work also investigated sensitivity of the results to the number of nuclides considered in the correction procedure. The results indicate that about 50-80 nuclides are necessary for the accurate reproduction of history effects, which still requires to track hundreds of nuclides in the depletion calculation if no simplifications and shortcuts are made. The reduced set of nuclides will significantly accelerate depletion calculation and could be used for accurate prediction of reactivity and power distribution. On the other hand, tracking detailed nuclide content (thousands of nuclides) would allow realistic modeling of decay heat and spent fuel activity without the need of any approximations. This application of the presented method is planned to be conducted in future work.

Further validation of the proposed method will include test cases with realistic geometry, burnable absorbers and partly inserted control rods. 


\section{References}

Bahadir, T., 2014. MODELING OF SHUTDOWN COOLING REACTIVITY EFFECTS WITH SIMULATE, in Proc.: PHYSOR 2014 - The Role of Reactor Physics Toward a Sustainable Future The Westin Miyako, Kyoto, Japan, September 28 - October 3, 2014.

Bahadir, T., Lindahl, S.-Ö., Palmtag, S.P., 2005. SIMULATE-4 multigroup nodal code with microscopic depletion model, in Proc.: Mathematics and Computation, Supercomputing, Reactor Physics and Nuclear and Biological Applications.

Baturin, D.M., Vygovskii, S.B., 2001. Taking Account of the Spectral History of Fuel Burnup during the Preparation of the Neutron-Physical Constants for VVÉR-1000 Fuel Assemblies. Atomic Energy 90, pp. 267-272.

Bilodid, I., Mittag, S., 2010. Use of the local Pu-239 concentration as an indicator of burnup spectral history in DYN3D. Annals of Nuclear Energy 37, pp. 1208-1213.

Bilodid, Y., Kotlyar, D., Margulis, M., Fridman, E., Shwageraus, E., 2015. Spectral history model in DYN3D: Verification against coupled Monte-Carlo thermal-hydraulic code BGCore. Annals of Nuclear Energy 81, pp. 34-40.

Bilodid, Y., Ovdiienko, I., Mittag, S., Kuchin, A., Khalimonchuk, V., Ieremenko, M., 2012. Assessment of spectral history influence on PWR and WWER core. Kerntechnik 77, pp. $278-285$.

Gomez-Torres, A.M., Sanchez-Espinoza, V.H., Ivanov, K., Macian-Juan, R., 2012. DYNSUB: A high fidelity coupled code system for the evaluation of local safety parameters - Part I: Development, implementation and verification. Annals of Nuclear Energy 48, pp. 108-122.

Gonchar, A.A., Rakhmanov, E.A., 1989. Equilibrium Distributions and Degree of Rational Approximation of Analytic Functions, Mat. Sb., 134, 306; see also Math. USSR Sb., 62, 2, 305 (translation).

Grundmann, U., Lucas, D., Rohde, U., 1995. Coupling of the thermohydraulic code ATHLET with the neutron kinetic core model DYN3D., in Proc.: Int. Conf. on Mathematics and Computations, Physics and Environmental Analysis, Vol. 1, Portland, USA.

Grundmann, U., Rohde, U., Mittag, S., Kliem, S., 2005. DYN3D version 3.2 - code for calculation of transients in light water reactors (LWR) with hexagonal or quadratic fuel elements - description of models and methods, HZDR, FZR-434, Dresden, Germany.

Holt, L., Rohde, U., Seidl, M., Schubert, A., Van Uffelen, P., Macián-Juan, R., 2015. Development of a general coupling interface for the fuel performance code TRANSURANUS - Tested with the reactor dynamics code DYN3D. Annals of Nuclear Energy 84, pp. 73-85.

Ida, T., Tahara, Y., 2000. Two Group Micro-Depletion Correction Model for 
ALPHA/PHOENIX-P/ANC Code System, in Proc.: PHYSOR 2000 Advances In Reactor Physics, and Mathematics and Computation Into the Next Millennium.

Kliem, S., Gommlich, A., Grahn, A., Rohde, U., Schutze, J., Frank, T., Gomez, A., Sanchez, V., 2011. Development of multi-physics code systems based on the reactor dynamics code DYN3D. Kerntechnik 76, pp. 160-165.

Kozmenkov, Y., Kliem, S., Grundmann, U., Rohde, U., Weiss, F.-P., 2007. Calculation of the VVER-1000 coolant transient benchmark using the coupled code systems DYN3D/RELAP5 and DYN3D/ATHLET. Nuclear Engineering and Design 237, pp. 1938-1951.

Leppänen, J., 2010. Performance of Woodcock delta-tracking in lattice physics applications using the Serpent Monte Carlo reactor physics burnup calculation code. Annals of Nuclear Energy 37, pp. 715-722.

Leppänen, J., 2009. Two practical methods for unionized energy grid construction in continuous-energy Monte Carlo neutron transport calculation. Annals of Nuclear Energy 36, pp. 878-885.

Leppänen, J., Pusa, M., Viitanen, T., Valtavirta, V., Kaltiaisenaho, T., 2015. The Serpent Monte Carlo code: Status, development and applications in 2013. Annals of Nuclear Energy 82, pp. 142-150.

Pusa, M., 2011. Rational approximations to the matrix exponential in burnup calculations. Nuclear Science and Engineering 169, pp. 155-167.

Pusa, M., Leppänen, J., 2010. Computing the matrix exponential in burnup calculations. Nuclear Science and Engineering 164, pp. 140-150. 